\title{
Generalized Variances Ratio Test for Comparing k Covariance Matrices from Dependent Normal Populations
}

Marcelo Angelo Cirillo

Federal University of Lavras, Brazil, macufla@gmail.com

Daniel Furtado Ferreira

Federal University of Lavras, Lavras Brazil, danielff@ufla.br

Thelma Sáfadi

Federal University of Lavras, Lavras Brazil, safadi@ufla.br

Eric Batista Ferreira

Federal University of Alfenas, Brazil, eric.ferreira@unifal-mg.edu.br

Follow this and additional works at: http://digitalcommons.wayne.edu/jmasm

Part of the Applied Statistics Commons, Social and Behavioral Sciences Commons, and the Statistical Theory Commons

\section{Recommended Citation}

Cirillo, Marcelo Angelo; Ferreira, Daniel Furtado; Sáfadi, Thelma; and Ferreira, Eric Batista (2010) "Generalized Variances Ratio Test for Comparing k Covariance Matrices from Dependent Normal Populations," Journal of Modern Applied Statistical Methods: Vol. 9 : Iss. 2 , Article 6.

DOI: $10.22237 /$ jmasm/1288584300

Available at: http://digitalcommons.wayne.edu/jmasm/vol9/iss2/6

This Regular Article is brought to you for free and open access by the Open Access Journals at DigitalCommons@WayneState. It has been accepted for inclusion in Journal of Modern Applied Statistical Methods by an authorized editor of DigitalCommons@WayneState. 


\title{
Generalized Variances Ratio Test for Comparing k Covariance Matrices from Dependent Normal Populations
}

\author{
Marcelo Angelo Cirillo Daniel Furtado Ferreira Thelma Sáfadi \\ Federal University of Lavras, \\ Lavras Brazil \\ Eric Batista Ferreira \\ Federal University of Alfenas, \\ Brazil
}

New tests based on the ratio of generalized variances are presented to compare covariance matrices from dependent normal populations. Monte Carlo simulation concluded that the tests considered controlled the Type I error, providing empirical probabilities that were consistent with the nominal level stipulated.

Key words: Dependent normal, bootstrap, variances generalized, power, type I error.

Introduction

Most statistical techniques assume that samples must be independent; however, practical situations where the samples come from dependent populations cannot be ignored. For example, a typical situation is a bioequivalence assay, the objective of which is to verify if a new drug presents effectiveness similar to a brandname drug. Thus, both drugs are applied to the same sample units, which are classified in two distinct groups and differentiated by the receiving order. The responses of such experiments are correlated and associated to a specific correlation structure.

A naturally appearing hypothesis in this type of experiment regards the equality of covariance matrices between a new drug and a brand-name drug (Wang, et al., 1999). The

Marcelo Cirillo is an Adjunct Lecturer III in the Exact Sciences Department. E-mail: macufla@gmail.com. Daniel Ferreira is an Associated Lecturer I in the Exact Sciences Department. Email: danielff@ufla.br. Thelma Sáfadi is an Adjunct Lecturer III in the Exact Sciences Department. Email: safadi@ufla.br. Eric Ferreira is an Adjunct Lecturer I. Email: eric.ferreira@unifal-mg.edu.br.
Bartlett test mentioned by O'Brien (1992) could not be used in this case, because its construction assumes independence of the samples. Due to the restriction of the current tests, the main goal of this study is to propose multivariate tests to verify the equality of covariance matrices considering dependence among multivariate observations along populations.

Another motivation justifying the need for a general test for the equality of covariance matrices of correlated data in time or space are the suppositions of analysis of variance and the Hotelling $\mathrm{T}^{2}$ test. It is required that the data submitted to multivariate analysis of variance have $\mathrm{p}$-variate normal residues, with null mean vector and constant covariance matrices. To check the assumption of constant covariances for $\mathrm{k}$ populations or treatments, a more general test is required. As noted, such tests do not exist or have limited properties for dependence structure situations.

Finney (1938) studied this problem considering the univariate case $(\mathrm{p}=1)$ and two populations $(\mathrm{k}=2)$ under a known correlation coefficient between the same variable in both populations. Pitman (1939) and Morgan (1939) proposed a likelihood ratio test for the case of $\mathrm{k}$ $=2$ populations, however with an unknown correlation matrix. Since that time, many authors have explored these results, all have considered 


\section{GENERALIZED VARIANCES RATIO TEST COMPARING K COVARIANCE MATRICES}

only the univariate case $(\mathrm{p}=1)$, although with different numbers of populations.

Roy and Potthoff (1958) concentrated on the bidimensional case, that is, $\mathrm{k}=2$ and $\mathrm{p} \geq$ 2 variables. However, they did not succeed in test construction. Jiang, et al. (1999) evidenced that the test considered by Roy and Potthoff (1958) presented deficiencies in the imposed presuppositions. Smith and Kshirsagar (1985) presented a likelihood ratio test to compare covariance matrices, coming from two dependent normal populations. However, the authors had not obtained the analytical expression of the maximum likelihood estimator under the null hypothesis. Due to some numerical problems in the maximization of the likelihood functions, the authors surrounded the problem using initial values such that the estimate of the covariance matrix was positive definite.

In a more general situation, represented by a number of populations $\mathrm{k} \geq 2$ and by a number of variables $p \geq 2$, Krishnaiah (1975) considered a test to compare two or more covariance matrices coming from dependent normal populations. This test was formalized under the assumption that the diagonals of the covariance matrices were equal; however, the main criticism to this test was that any restriction or assumption was made for the dependence structure between those matrices.

Jiang, et al. (1999) used Monte Carlo simulation to evaluate some tests based on a likelihood ratio used in the comparison of covariance matrices of dependent normal populations. The differentiation between each test was made under different corrections in the degrees of freedom as proposed by several authors. It was such that - for each correction new statistics had arisen. Results were restricted to the bidimensional case, and the extension of these tests for $p$ dimensions became impracticable in the face of the numerical problem in the likelihood maximization. Because finding a general test based upon the likelihood ratio to compare $\mathrm{k}$ dependent population covariances is a difficult task, the bootstrap method can be used (Manly, 1997). Bootstrapping is typically used to round problems for which an analytical solution is not straightforward. Due to the dependency between populations, Hall, et al., (1995) recommend the use of implicit resample in bootstrap, which must be done in blocks. This article proposes multivariate tests for comparing covariance matrices from $\mathrm{k}$ dependent multivariate normal populations, as well as studying their power and type I error probability.

\section{Methodology}

The multivariate tests considered in this article have been constructed considering the multivariate observation represented by the vector of random variables $\underset{\sim}{X}$, where each component $\underset{\sim}{\mathrm{X}}, \ldots, \underset{\sim}{\mathrm{t}}$ is composed of $\mathrm{p}$ dimensional vectors of random variables $\underset{\sim j}{X}=\left(X_{j 1}, \ldots, X_{j p}\right)^{t}, j=1 \ldots, k$, where $k$ refers to the total number of populations and $p$ to the number of variables. The vector $\underset{\sim}{X}$ is then a pkdimensional random variable from a multivariate normal distribution, $\mathrm{N}_{\mathrm{pk}}(\underset{\sim}{\underset{\sim}{\mu}, \Sigma)}$, whose parameters are defined as:

$$
{\underset{\sim}{\mathrm{pk} \times 1}}_{\mathrm{a}}=\left[\begin{array}{c}
\underset{\sim}{\mu_{1}} \\
\underset{\sim}{\mu_{2}} \\
\vdots \\
\underset{\sim}{\mu_{\mathrm{k}}}
\end{array}\right]
$$

and

$$
\Sigma_{\mathrm{pk} \times \mathrm{pk}}=\left(\begin{array}{ccc}
\Sigma_{11} & \ldots & \Sigma_{1 \mathrm{k}} \\
\vdots & \ddots & \vdots \\
\Sigma_{\mathrm{k} 1} & \cdots & \Sigma_{\mathrm{kk}}
\end{array}\right)
$$

The off diagonal elements indicate non-null covariances between populations because independence was not assumed. Each element in the diagonal of $\Sigma$ represents the covariance matrix of the $\mathrm{j}^{\text {th }}$ population. The hypothesis of interest is: $\mathrm{H}_{0}: \Sigma_{11}=\Sigma_{22}=\ldots=\Sigma_{\mathrm{kk}}$ versus $\mathrm{H}_{1}$ : At least one covariance matrix $\Sigma_{\mathrm{jj}}$ differs from the others.

Statistics of the proposed tests were specified by the function of the ratio of generalized variances, as follows: 


\section{CIRILLO, FERREIRA, SÁFADI \& FERREIRA}

$$
\begin{aligned}
& \lambda_{1(b)}=\frac{\max _{\mathrm{j}}\left(\left|\mathrm{S}_{\mathrm{jj}}\right|\right)}{\min _{\mathrm{j}}\left(\left|\mathrm{S}_{\mathrm{jj}}\right|\right)} ; \\
& \lambda_{2(\mathrm{~b})}=\frac{\max _{\mathrm{j}}\left(\operatorname{Trace}\left[\mathrm{S}_{\mathrm{jj}}\right]\right)}{\min _{\mathrm{j}}\left(\operatorname{Trace}\left[\mathrm{S}_{\mathrm{jj}}\right]\right)}
\end{aligned}
$$

where $S_{\mathrm{jj}}$ are estimators of the sum of squares and products matrices. Each test was differentiated by the criterion used in the composition of the ratio, namely determinant or trace. Estimators of the sum of squares and products matrices of the $\mathrm{j}^{\text {th }}$ population $(\mathrm{j}=1,2, \ldots$, k) were only considered after the imposition of $\mathrm{H}_{0}$ through the bootstrap method (Figure 1).

After defining the test statistics, the multivariate samples considering equicorrelation structure were generated in order to evaluate the performance of the new tests. Thus, specifying the matrix $\Sigma$, proceeded as follows. A global (population) correlation matrix $\mathrm{R}_{\mathrm{b}}$, where each block element in the diagonal represents a correlation structure referring to the $\mathrm{j}^{\text {th }}$ population (the area delimited by hatched lines) is given by:

$\mathrm{R}_{\mathrm{b}}=\left[\begin{array}{cccccccccc}1 & \rho & \cdots & \rho & \rho & \rho & \rho & \cdots & \cdots & \rho \\ \rho & 1 & \vdots & \vdots & \ddots & \ddots & \ddots & \ddots & \ddots & \rho \\ \vdots & \vdots & \ddots & \vdots & \ddots & \ddots & \ddots & \ddots & \ddots & \rho \\ \rho & \cdots & \cdots & 1 & \ddots & \ddots & \ddots & \ddots & \ddots & \rho \\ \rho & \ddots & \ddots & \ddots & \ddots & \ddots & \ddots & \ddots & \ddots & \rho \\ \rho & \ddots & \ddots & \ddots & \ddots & \ddots & 1 & \rho & \cdots & \rho \\ \vdots & \ddots & \ddots & \ddots & \ddots & \ddots & \rho & 1 & \vdots & \vdots \\ \vdots & \ddots & \ddots & \ddots & \ddots & \ddots & \vdots & \vdots & \ddots & \vdots \\ \rho & \rho & \rho & \rho & \rho & \cdots & \rho & \cdots & \cdots & 1\end{array}\right]$.

The global covariance matrix is obtained from the following relation:

$$
\Sigma^{*}=\mathrm{V}^{\frac{1}{2}} \mathrm{R}_{\mathrm{b}} \mathrm{V}^{\frac{1}{2}}
$$

where $\mathrm{V}^{\frac{1}{2}}$ is a diagonal matrix of the population standard deviations which are all equal to 1 , without loss of generality.

After defining, samples were generated using the Monte Carlo method; an algorithm was developed using $\mathrm{R}$ software version 2.6.2 assuming multivariate normal distribution $\mathrm{N}_{\mathrm{pk}}\left(\underset{\sim}{0}, \Sigma^{*}\right)$. The algorithm first evaluated the Type I error rates of the related tests when applied to samples simulated under the null hypothesis $\mathrm{H}_{0}$. Power was not measured at this stage because all diagonal block elements of $\Sigma^{*}$ were considered equal.

Power rates were evaluated for those tests applied to samples simulated under the alternative hypothesis. The global population covariance matrix should be defined in such a way that each population matrix (diagonal blocks) would have to obey the heterogeneity settled in an intended value $\delta$. In both situations, under null and alternative hypotheses, those matrices were evaluated in situations of low and high correlation, originated from structures represented by parametric values $\rho$ fixed in 0.2 and 0.8 .

Under $\mathrm{H}_{1}$, the $\Sigma_{\mathrm{g}}$ matrix was defined as:

$$
\Sigma_{\mathrm{g}}=\mathrm{V}^{\frac{1}{2}} \mathrm{R}_{\mathrm{b}} \mathrm{V}^{\frac{1}{2}}
$$

where

$$
\begin{aligned}
& \mathrm{V}= \\
& \operatorname{diag}\left[11 \ldots 1: \sqrt[2 p]{\mathrm{d}_{2}} \sqrt[2 p]{\mathrm{d}_{2}} \ldots \sqrt[2 p]{\mathrm{d}_{2}}: \ldots: \sqrt[2 p]{\mathrm{d}_{\mathrm{k}}} \ldots \sqrt[2 p]{\mathrm{d}_{\mathrm{k}}}\right]
\end{aligned}
$$

Each block (6) was p-dimensional and

$$
d_{j}=\sqrt[p]{1+\frac{(j-1) \times(\delta-1)}{k-1}}
$$

for $\mathrm{j}=1,2, \ldots, \mathrm{k}$, and $\delta=2,4,8,16$.

After defining the covariance matrix parameters $\Sigma^{*}(\delta=1)$ and $\sum_{\mathrm{g}}(\delta>1)$, multivariate sample observations used in the evaluation of the considered tests were simulated. The $\mathrm{N}$ vector set generated formed the matrix of sample data: 


$$
\mathrm{X}=\left[\begin{array}{c}
\underset{\sim}{\mathrm{X}} \\
\vdots \\
\mathrm{X}_{\mathrm{N}}^{\mathrm{t}}
\end{array}\right],
$$

where $\underset{\sim l}{\mathrm{X}}$ is a $\mathrm{pk} \times 1$ vector and $\mathrm{N}$ is the sample size.

The construction the matrix was carried through using the vector of observations coming from the joint distribution of the $\mathrm{k}$ populations, generated according to the multivariate normal distribution,

$$
\underset{\sim}{\mathrm{X}}=\underset{\sim}{\mathrm{FZ}} \underset{\sim}{\operatorname{\sim }}+\underset{\sim}{\mu}(\ell=1,2, \ldots, \mathrm{N}),
$$

where $\mathrm{F}$ is the Cholesky factor (Bock, 1975) of the population covariance matrix $\Sigma_{\mathrm{g}}$ or $\Sigma^{*}$; and $\underset{\sim}{\mathrm{Z}}$ is a $\mathrm{kp} \times 1$ vector of independent standard normal variables, generated by the inversion of the distribution function of the standard univariate normal in a random point $\mathrm{U}$, $\mathrm{U} \sim \mathrm{U}[0,1]$.

After obtaining the multivariate normal samples, the vector of sample means of $\mathrm{kp}$ variables was estimated by $\underset{\sim}{\bar{X}}=\left(\bar{\sim}_{1}^{\mathrm{X}}, \bar{\sim}_{2}^{\mathrm{X}}, \cdots,{\underset{\sim}{\mathrm{X}}}_{\mathrm{t}}^{\mathrm{t}}\right)^{\mathrm{t}}$, where $(\mathrm{j}=1, \ldots, \mathrm{k})$. The deviations of the vector of means were then computed in order to allow no influence of possible different averages between the $\mathrm{k}$ populations on the estimators of the covariance matrices. Thus, the inference was made considering the matrix of deviations $\mathrm{X}_{\mathrm{d}}$, defined as:

$$
\mathrm{X}_{\mathrm{d}}=\mathrm{X}-\underset{\sim}{1} \overline{\mathrm{X}}^{\mathrm{t}}
$$

where $\underset{\sim}{1}$ is a vector $\mathrm{N} \times 1$. The sum of squares and products matrix was estimated by

$$
\mathrm{S}=\left(\mathrm{X}_{\mathrm{d}}\right)^{\mathrm{t}} \mathrm{Q} \mathrm{X}_{\mathrm{d}}
$$

where the projection matrix is given by

$$
\mathrm{Q}=\mathrm{I}-\frac{\underset{\sim}{1} \stackrel{\sim}{\mathrm{t}}^{\mathrm{n}}}{\mathrm{n}}
$$

where $\underset{\sim}{1}$ is a vector of 1 's $(\mathrm{N} \times 1)$ and $\mathrm{I}(\mathrm{N} \times \mathrm{N})$ is an identity matrix.

Given the random sample $X_{d}, 1,000$ resamples were drawn. In each resample a new bootstrap sample $X_{d b}$, was obtained, of which the matrix of sum of squares and products was estimated and named $\mathrm{S}_{\mathrm{b}}^{*},(\mathrm{~b}=1, \ldots, 1,000)$. The elements of the diagonal blocks $\hat{S}_{b(j j)}^{*}(j=1,2$, $\ldots, \mathrm{k})$ of dimensionality $(\mathrm{p} \times \mathrm{p})$ represent the estimators of the population sum of squares and products matrices, used to determine the statistics based on the generalized variances ratio. In each resample, values $\lambda_{1(\mathrm{~b})}$ and $\lambda_{2(\mathrm{~b})}$ were computed and compared with $\lambda_{1}$ and $\lambda_{2}$, obtained in the original sample of the Monte Carlo simulation. The critical region for the considered tests was constructed on the empirical distribution of the values of the statistics $\lambda_{1(\mathrm{~b})}$ and $\lambda_{2(\mathrm{~b}) \text {. }}$

The critical stage of this procedure was setting the null hypothesis of equality of the population covariance matrices, surrounding all restrictions of the numerical methods of likelihood function maximization. The bootstrap method (Figure 1) considers as randomization unit the multivariate sample unit (SU) of each population considering $\mathrm{p}$ variables, thus characterizing $\mathrm{H}_{0}$, which was set considering the dependence between the variables of all kpopulations.

For each situation designed by the combination of the number of variables $(\mathrm{p}=2$, $3,8)$, number of populations $(\mathrm{k}=2,8,12)$, sample size $(\mathrm{N}=20,50,100)$ and nominal values $1 \%$ and $5 \%$, the empirical probabilities were computing by the times that the values of the statistics $\lambda_{1(\mathrm{~b})}$ and $\lambda_{2(\mathrm{~b})}$ were greater than or equal to the values $\lambda_{1}$ e $\lambda_{2}$ respectively. These values were obtained in the original sample in relation to the total number of bootstrap. The empirical type I error rates and power had been computed considering the proportion of times that $\mathrm{H}_{0}$ was rejected by the nominal levels of $1 \%$ and considered $5 \%$ under $\mathrm{H}_{0}$ and $\mathrm{H}_{1}$, respectively. 


\section{CIRILLO, FERREIRA, SÁFADI \& FERREIRA}

Figure 1: Bootstrap Process Used to Estimate the Matrices of Sum of Squares and Products Coming from Dependent Multivariate Normal Populations

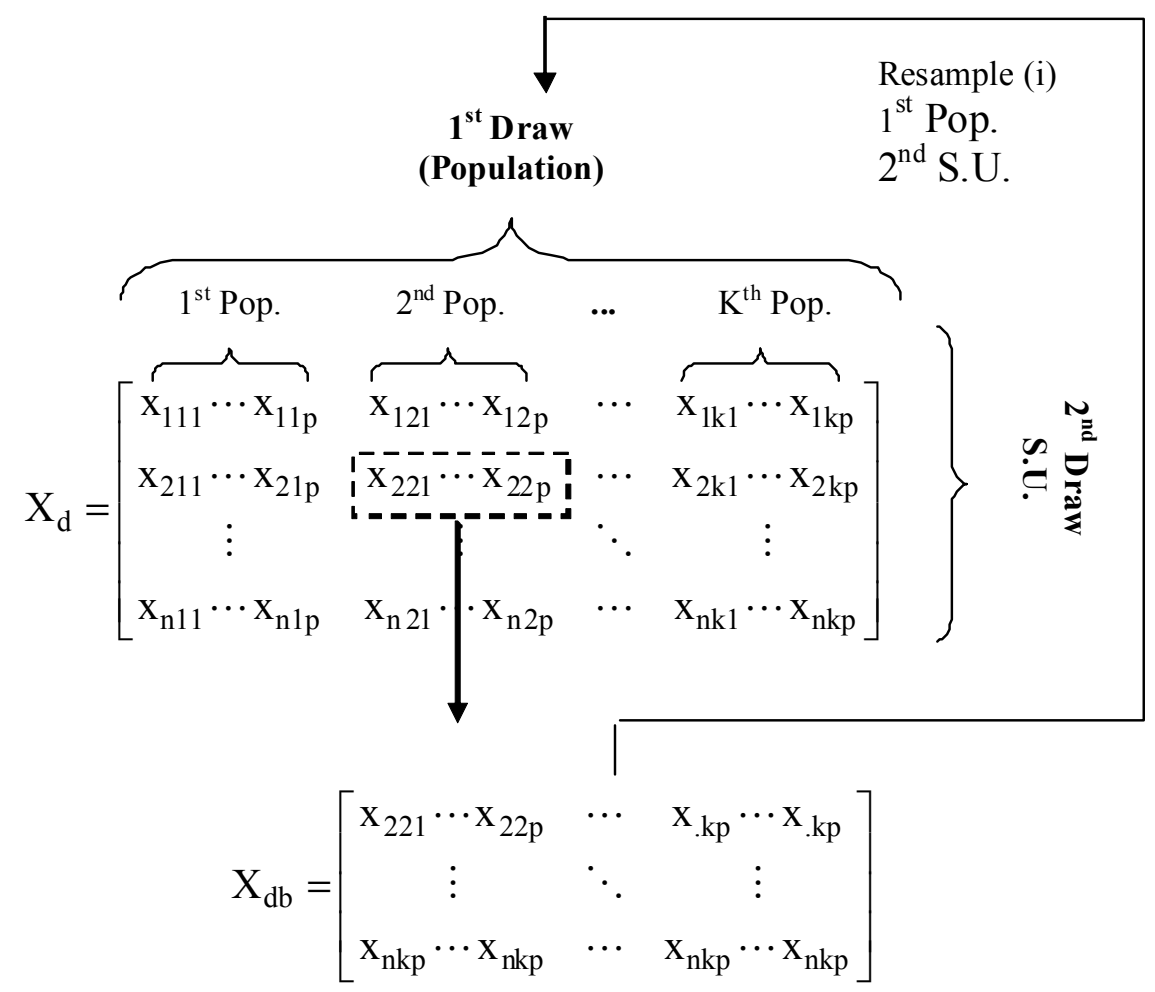

Results

Probabilities of Type I Error

Using a $95 \%$ confidence interval for the adopted nominal level, it can be inferred that the test was conservative if the value of the probability was less than the inferior limit. However, probability values contained between the interval limits demonstrated that the tests had provided effective control of type I error rates, that is, they have exact size. Table 2 contains the empirical Type I error rates, where was used the generalized variances obtained from the ratio of determinants.

Results in Table 2 show that the test based on the ratio of determinants submitted to low covariances $(\rho=0.20)$ controlled the Type I error with probabilities equal to or less than the nominal level set at $5 \%$ in almost all the evaluated situations. The exception occurred when the test was submitted to a high number of populations and had a small sample $(\mathrm{N}=20)$. Increasing the value of the global correlation for $\rho=0.80$ implied greater averages in the covariances and it was verified that the test was conservative in general. Results in Table 3 show the probabilities of the test considering the ratio of total variances (trace) evaluated with the same situations as that of the previous test.

In general, when using the ratio of total variances statistic, conservative results were obtained for those samples submitted with low global correlation $(\rho=0.20)$. In the high correlation cases $(\rho=0.80)$, the results of the test remain conservative, despite using determinant or trace.

Comparing the results of the generalized variances ratio tests presented in this article with the likelihood ratio tests considered by Bartlett (1937), Box (1949) and Krishnaiah (1975), it can be affirmed that the likelihood ratio tests are not adequate to compare dependent multivariate populations. Such affirmation is based on the fact that these tests have been compared with results presented by Jiang, et al. (1999) who 
Table 2: Type I Error Rates for the Situations of Low and High Correlation, Evaluated In the Combinations of Number of Populations (k), Number of Variables (p) Considering the Test Defined by the Determinant Ratio

\begin{tabular}{|c|c|c|c|c|c|c|}
\cline { 2 - 7 } & \multicolumn{5}{c|}{$\mathrm{k}=2$} & \multicolumn{2}{c|}{$\mathrm{k}=8$} & $\mathrm{k}=12$ \\
\cline { 2 - 7 } & $\mathrm{p}=2$ & $\mathrm{p}=3$ & $\mathrm{p}=8$ & $\mathrm{p}=2$ & $\mathrm{p}=3$ & $\mathrm{p}=8$ \\
\hline $\mathrm{N}$ & 0.039 & 0.04 & $0.029^{*}$ & 0.045 & $0.016^{*}$ & $0.036^{*}$ \\
\hline 20 & 0.045 & 0.040 & 0.038 & 0.039 & 0.043 & 0.037 \\
\hline 50 & 0.037 & 0.039 & 0.047 & 0.046 & 0.050 & 0.048 \\
\hline 100 & \multicolumn{6}{|c|}{$\rho=0.80$} \\
\hline $\mathrm{N}$ & $0.016^{*}$ & $0.015^{*}$ & $0.011^{*}$ & $0.011^{*}$ & $0.014^{*}$ & $0.018^{*}$ \\
\hline 20 & $0.006^{*}$ & $0.014^{*}$ & $0.004^{*}$ & $0.005^{*}$ & $0.036^{*}$ & 0.039 \\
\hline 50 & $0.004^{*}$ & $0.020^{*}$ & $0.006^{*}$ & $0.006^{*}$ & 0.052 & $0.036^{*}$ \\
\hline 100 & &
\end{tabular}

*empirical probabilities under the lower limit of the $95 \%$ confidence interval $(0.037 ; 0.065)$

Table 3: Type I Error Rates for the Situations of Low and High Correlation Evaluated In the Combinations of Number of Populations (k), Number of Variables (p) Considering the Test Defined By the Ratio of Traces

\begin{tabular}{|c|c|c|c|c|c|c|}
\cline { 2 - 7 } & \multicolumn{5}{c|}{$\mathrm{k}=2$} & \multicolumn{2}{c|}{$\mathrm{k}=8$} & $\mathrm{k}=12$ \\
\cline { 2 - 7 } & $\mathrm{p}=2$ & $\mathrm{p}=3$ & $\mathrm{p}=8$ & $\mathrm{p}=2$ & $\mathrm{p}=3$ & $\mathrm{p}=8$ \\
\hline $\mathrm{N}$ & 0.042 & 0.044 & $0.032^{*}$ & $0.037^{*}$ & $0.031^{*}$ & $0.035^{*}$ \\
\hline 20 & 0.039 & $0.035^{*}$ & $0.032^{*}$ & $0.046^{*}$ & 0.030 & $0.031^{*}$ \\
\hline 50 & 0.040 & $0.036^{*}$ & $0.031^{*}$ & 0.037 & $0.034^{*}$ & 0.043 \\
\hline 100 & \multicolumn{6}{|c|}{$\rho=0.80$} \\
\hline $\mathrm{N}$ & $0.000^{*}$ & $0.000^{*}$ & $0.000^{*}$ & $0.001^{*}$ & $0.006^{*}$ & $0.001^{*}$ \\
\hline 20 & $0.000^{*}$ & $0.000^{*}$ & $0.000^{*}$ & $0.001^{*}$ & $0.000^{*}$ & $0.001^{*}$ \\
\hline 50 & $0.000^{*}$ & $0.000^{*}$ & $0.000^{*}$ & $0.001^{*}$ & $0.002^{*}$ & $0.000^{*}$ \\
\hline 100 & 0
\end{tabular}

"empirical probabilities under the lower limit of the $95 \%$ confidence interval $(0.037 ; 0.065)$

used Monte Carlo simulations to verify that, in general, the likelihood ratio tests did not control type I error when $\mathrm{N}=10,15,20,25,50,75$ and 100 under several correlation structures. However, asymptotic tests considered by the authors did control type I error for samples greater than $50(\mathrm{~N}>50)$ with probabilities close to the nominal level.
For the tests evaluated herein, it was observed that, for samples sizes smaller than 50 $(\mathrm{N}<50)$, the tests were conservative under correlation $\rho=0.80$. It is noteworthy that results obtained by other authors were related to bivariate populations only. This limitation was due to the maximization of the likelihood functions problem. Thus, for larger numbers of 


\section{CIRILLO, FERREIRA, SÁFADI \& FERREIRA}

populations and variables no results exist in the literature, regarding means of the likelihood theory that could be compared with the results of this present work. Results shown in Table 4 were obtained under the same configurations previously evaluated, but with the nominal level set to $1 \%$. However, $\mathrm{k}=8$ populations on $\mathrm{p}=12$ variables were evaluated in particular, because this represents an extreme case and because cases considering $\mathrm{k}>2$ could not be found in the literature.

Similarly, by estimating a $95 \%$ confidence interval for this nominal level it can be verified whether or not the test was conservative. It was observed that the results for a $1 \%$ level of significance had the same pattern as results at the $5 \%$ level. Due to the similarity in results of the type I error rates, it is expected that the power function would be similar and coherent for both nominal levels $1 \%$ and $5 \%$. It is worth noting that this similarity to the pattern of type I error rates between $1 \%$ and $5 \%$ also was observed in other configurations evaluated in $\mathrm{k}$ variables and $\mathrm{p}$ populations, thus, not all results are shown.
Power of the Multivariate Tests for Comparing Covariance Matrices of $\mathrm{k}$ Dependent Normal Populations

Power results corresponded to the empirical probabilities, which were obtained under the same configurations evaluated in the control of type I error rate discussed previously using the bootstrap method (see Figure 1). Results shown in Table 5 consider low global correlation $(\rho=0.20)$.

Analyzing the results in Table 5, it is observed that by increasing the degree of heterogeneity $(\delta)$ in all evaluated situations the power of the test suffers incrementally. However, for sample sizes $\mathrm{N}=50$ and greater, cases of $\delta=8$ were similar to situations where $\delta$ $=16$. This suggests that - for any degree of heterogeneity $(\delta>8)$ between covariance matrices - the considered test was powerful when the population covariances had relatively low correlation.

An interesting result can be observed in power evaluation as the number of populations (k) rises. The power of the test presents few oscillations under a degree of heterogeneity $(\delta)$

Table 4: Probabilities of Type I Error Considering the Generalized Variance Given By the Ratio of Determinants and the Ratio of Traces In the Two Evaluated Global

Correlations with Nominal Significance Level $1 \%, k=8$ and $p=12$

\begin{tabular}{|c|c|c|}
\hline \multicolumn{3}{|c|}{ Ratio of Determinants } \\
\hline $\mathrm{N}$ & $\rho=0.20$ & $\rho=0.80$ \\
\hline 20 & 0.0116 & $0.0033^{*}$ \\
\hline 50 & 0.0050 & 0.0066 \\
\hline 100 & 0.0150 & 0.0133 \\
\hline \multicolumn{3}{|c|}{ Ratio of Traces } \\
\hline $\mathrm{N}$ & $\rho=0.20$ & $\rho=0.80$ \\
\hline 20 & 0.0100 & $0.0000^{*}$ \\
\hline 50 & $0.0016^{*}$ & $0.0000^{*}$ \\
\hline 100 & 0.0083 & $0.0000^{*}$ \\
\hline
\end{tabular}




\section{GENERALIZED VARIANCES RATIO TEST COMPARING K COVARIANCE MATRICES}

greater than 8 and sample sizes greater than 50 , but does not hold for the case where a high number of variables are considered $(\mathrm{p}=12)$. Regarding performance, when the number of variables (p) increases for a settled number of populations $(\mathrm{k})$ and when bivariate populations $(\mathrm{k}=2)$ are considered, the test becomes more sensitive, thus decreasing its power. Under low heterogeneity $(\delta)$, the test showed discrepant results for small samples $(\mathrm{N}=20)$. Clearly, for a great number of variables $(p=8)$, the reduction of power was even more drastic. With respect to $\mathrm{k}=8$ populations, the number of variables caused less reduction of power, considering a maximum degree of heterogeneity of this study $(\delta=16)$.
Regarding the effect of increasing the sample size $(\mathrm{N})$, the power for cases with small samples was small, what agrees with empirical Type I error rate probabilities (Table 2). In such situations the test was revealed to be conservative. Note such deficiency of power, caused by the conservative property of the test (Table 2), does not invalidate it. Tests comparing $\mathrm{k}$ dependent population covariance matrices for many populations do not exist in the literature. Results shown in Table 6 emphasize the performance of the generalized variances test as represented by the determinants ratio under a global correlation $(\rho=0.8)$ and considering the same situations evaluated previously.

Table 5: Power Empirical Values for the Bootstrap Generalized Likelihood Ratio Test for Different Sample Sizes (n), Numbers of Populations (k), Variables (p),

Degrees of Heterogeneity $(\delta)$ Under Low Global Correlation $(\rho=0.20)$

\begin{tabular}{|c|c|c|c|c|c|c|}
\hline $\mathrm{N}$ & $\mathrm{k}=2$ & $\mathrm{k}=2$ & $\mathrm{k}=2$ & $\mathrm{k}=8$ & $\mathrm{k}=8$ & $\mathrm{k}=8$ \\
$\mathrm{p}=2$ & $\mathrm{p}=3$ & $\mathrm{p}=8$ & $\mathrm{p}=2$ & $\mathrm{p}=3$ & $\mathrm{p}=12$ \\
\hline \multicolumn{7}{|c|}{$\delta=2$} \\
\hline 20 & 0.150 & 0.080 & 0.050 & 0.090 & 0.090 & 0.070 \\
\hline 50 & 0.370 & 0.230 & 0.220 & 0.200 & 0.180 & 0.080 \\
\hline 100 & 0.610 & 0.650 & 0.470 & 0.430 & 0.270 & 0.120 \\
\hline \multicolumn{7}{|c|}{$\delta=4$} \\
\hline 20 & 0.490 & 0.320 & 0.120 & 0.300 & 0.180 & 0.100 \\
\hline 50 & 0.900 & 0.790 & 0.820 & 0.820 & 0.550 & 0.190 \\
\hline 100 & 0.970 & 0.980 & 0.950 & 0.980 & 0.930 & 0.520 \\
\hline \multicolumn{7}{|c|}{$\delta=8$} \\
\hline 20 & 0.810 & 0.600 & 0.150 & 0.710 & 0.430 & 0.090 \\
\hline 50 & 0.970 & 1.000 & 0.950 & 0.980 & 0.910 & 0.470 \\
\hline 100 & 0.980 & 1.000 & 0.950 & 0.980 & 1.000 & 0.900 \\
\hline \multicolumn{7}{|c|}{$\delta=16$} \\
\hline 20 & 0.95 & 0.890 & 0.220 & 0.950 & 0.760 & 0.180 \\
\hline 50 & 0.98 & 1.000 & 0.950 & 0.980 & 1.000 & 0.730 \\
\hline 100 & 0.980 & 1.000 & 0.950 & 0.980 & 1.000 & 0.990 \\
\hline
\end{tabular}




\section{CIRILLO, FERREIRA, SÁFADI \& FERREIRA}

Table 6: Power Values for the Bootstrap Generalized Likelihood Ratio Test for Different Sample Sizes (n), Numbers of Populations (k), Variables (p), Degrees of

Heterogeneity $(\delta)$ Under Low Global Correlation $(\rho=0.80)$

\begin{tabular}{|c|c|c|c|c|c|c|}
\hline & $\mathrm{k}=2$ & $\mathrm{k}=2$ & $\mathrm{k}=2$ & $\mathrm{k}=8$ & $\mathrm{k}=8$ & $\mathrm{k}=8$ \\
$\mathrm{~N}$ & $\mathrm{p}=2$ & $\mathrm{p}=3$ & $\mathrm{p}=8$ & $\mathrm{p}=2$ & $\mathrm{p}=3$ & $\mathrm{p}=12$ \\
\hline \multicolumn{7}{|c|}{$\delta=2$} \\
\hline 20 & 0.110 & 0.080 & 0.030 & 0.020 & 0.020 & 0.050 \\
\hline 50 & 0.380 & 0.230 & 0.100 & 0.180 & 0.030 & 0.060 \\
\hline 100 & 0.730 & 0.590 & 0.330 & 0.410 & 0.110 & 0.100 \\
\hline \multicolumn{7}{|c|}{$\delta=4$} \\
\hline 20 & 0.500 & 0.370 & 0.070 & 0.170 & 0.040 & 0.070 \\
\hline 50 & 0.930 & 0.780 & 0.720 & 0.760 & 0.200 & 0.220 \\
\hline 100 & 0.980 & 0.950 & 0.700 & 0.990 & 0.500 & 0.580 \\
\hline \multicolumn{7}{|c|}{$\delta=8$} \\
\hline 20 & 0.890 & 0.600 & 0.090 & 0.580 & 0.090 & 0.110 \\
\hline 50 & 0.990 & 0.980 & 0.950 & 0.960 & 0.450 & 0.480 \\
\hline 100 & 0.990 & 0.980 & 0.980 & 1.000 & 0.900 & 0.930 \\
\hline \multicolumn{7}{|c|}{$\delta=16$} \\
\hline 20 & 1.000 & 0.870 & 0.210 & 0.920 & 0.180 & 0.200 \\
\hline 50 & 1.000 & 1.000 & 0.950 & 1.000 & 0.800 & 0.840 \\
\hline 100 & 1.000 & 1.000 & 0.980 & 1.000 & 0.980 & 0.990 \\
\hline
\end{tabular}

Comparing results in Tables 5 and 6 , observe that an increment of the degree of heterogeneity $(\delta)$ yields an increment of the power values. However, this increment was small, since for $\mathrm{N}=20,50$ and degree of heterogeneity $\delta=4$, the test remains not so powerful.

In a general manner, the number of populations $(\mathrm{k})$ is related to a reduction of power, retaining the same highlighted properties of when the population covariance matrices presented, in average, low correlation (Table 5). However, in comparison to results shown in Table 6, it is suggested that increasing the global correlation yields an even greater reduction in power. Therefore, it may be concluded that increasing the number of populations $(\mathrm{k})$ where population covariances present high correlations results in a great loss of power. In turn, when the number of variables $(p)$ is increased with a set number of populations $(\mathrm{k})$, the test retained the same properties. The increment of the global correlation from $\rho=0.20$ for $\rho=0.80$ also did not affect the power of the test when the number of variables ( $p$ ) was increased for a set number of populations (k).

Regarding the sample size, results shown in Table 6 agree with previous results. It is advisable to use the considered test to deal with small samples when comparing bivariate populations $(\mathrm{k}=2)$ with a degree of heterogeneity greater than 8 . Such a test can be used for other cases; however, exploratory studies of the populations must be done.

\section{Conclusion}

Generalized variances tests controlled type I error according to a set nominal level. Tests based on the ratio of traces, in general, provided more conservative results. The simulation results clearly demonstrated that the procedure based on the determinant could more effectively control 


\section{GENERALIZED VARIANCES RATIO TEST COMPARING K COVARIANCE MATRICES}

the type I error rate to $\alpha$, particularly when the off-diagonal elements of $\mathrm{R}_{\mathrm{j}}$, the correlation matrix corresponding to $\Sigma_{\mathrm{j}}$, are small. Power of the generalized variances tests was reduced by increasing the number of variables and populations in both global correlations evaluated.

\section{References}

Bock, R. D. (1975). Multivariate Statistical Methods in Behavioral Research, New York: McGraw-Hill.

Bartlett, M. S. (1937). Properties of sufficiency and statistical tests. Proceedings of the Royal Statistical Society, 160, 268-282.

Box, G. E. P. (1949). A general distribution theory for a class of likelihood criteria. Biometrika, 36, 317-346.

Finney, D. J. (1938). The distribution of the ratio of estimates of the two variances in a sample from a normal bivariate population. Biometrika, 30, 190-192.

Hall, P., Horowitz,J. L., \& Jiang, B. (1995). On blocking rules for the bootstrap with dependent data. Biometrika, 82, 561-574.

Jiang, G., Sarkar, K. S., \& Hsuan, F. (1999). A likelihood ratio test and its modifications for the homogeneity of the covariance matrices of dependent multivariate normals. Journal of Statistical Planning and Inference, 81, 95-111.

Krishnaiah, P. R. (1975). Tests for the equality of the matrices covariance of correlated multivariate normal populations. In J. N. Srivastava (Ed.), A survey statistical design and linear models, 355-366. Amsterdam: NorthHolland.
Manly, B. F. J. (1997). Randomization Bootstrap and Monte Carlo Methods in Biology, New York: Chapman \& Hall.

Morgan, W. A. (1939). A test for the significance of the difference between the two variances in a sample from a normal bivariate population. Biometrika, 31, 9-12.

O'Brien, C. O. P. (1992). Robust Procedures for Testing Equality of Covariance Matrices. Biometrics, 48, 819-827.

Pitman, E. J. G. (1939). A note on normal correlation. Biometrika, 31, 9-12.

R Development Core Team (2008). R: A language and environment for statistical computing. $\mathrm{R}$ Foundation for Statistical Computing, Vienna, Austria. ISBN 3-90005107-0, URL http://www.R-project.org.

Roy, S. N., \& Potthoff, R. F. (1958). Confidence bounds on vector analogues of the ratio of means and the ratio of variances for two correlated normal variates and some associated tests. Annals of Mathematical Statistics, 29, 829841.

Smith, P. L., \& Kshirsagar, A. M. (1985). Testing for the equality of the variancecovariance matrices of two jointly normal vector variables. Biometrika, 27, 581-589.

Wang, W., Hwang, J. T. G., \& Dasgupta, A. (1999). Statistical tests for multivariate bioequivalence, Biometrika, 86, 395-402. 